OPEN ACCESS

Edited by:

Berthold Langguth,

University of Regensburg,

Germany

Reviewed by:

Juan Domènech,

University of Barcelona, Spain

Miguel J. A. Láinez,

Hospital Clínico Universitario de

Valencia, Spain

${ }^{*}$ Correspondence:

Arnaud J. Noreña

arnaud.norena@univ-amu.fr

Specialty section:

This article was submitted

to Neuro-Otology,

a section of the journal

Frontiers in Neurology

Received: 23 June 2017 Accepted: 03 August 2017

Published: 16 August 2017

Citation:

Londero A, Charpentier N, Ponsot D, Fournier $P$, Pezard $L$ and Noreña $A J$

(2017) A Case of Acoustic Shock with Post-trauma TrigeminalAutonomic Activation.

Front. Neurol. 8:420.

doi: 10.3389/fneur.2017.00420

\section{A Case of Acoustic Shock with Post-trauma Trigeminal-Autonomic Activation}

\author{
Alain Londero', Nicolas Charpentier ${ }^{2}$, Damien Ponsot ${ }^{3}$, Philippe Fournier ${ }^{4}$, Laurent Pezard ${ }^{4}$ \\ and Arnaud J. Noreña ${ }^{* *}$ \\ ${ }^{1}$ Service ORL et CCF, Hôpital Européen G. Pompidou, Paris, France, ${ }^{2}$ Faculté de médecine de Nancy, Université de \\ Lorraine, Nancy, France, ${ }^{3}$ Lycée Germaine Tillion, Académie de Lyon, Sain-Bel, France, ${ }^{4}$ Laboratoire Neurosciences \\ Intégratives et Adaptatives, UMR CNRS 7260, Fédération 3C, Aix-Marseille Université, Marseille, France
}

This study reports the case of an acoustic shock injury (ASI), which did not result in a significant hearing loss, but was followed by manifold chronic symptoms both within (tinnitus, otalgia, tingling in the ear, tension in the ear, and red tympanum) and outside the ears (blocked nose, pain in the neck/temporal region). We suggest that these symptoms may result from a loop involving injury to middle ear muscles, peripheral inflammatory processes, activation and sensitization of the trigeminal nerve, the autonomic nervous system, and central feedbacks. The pathophysiology of this ASI is reminiscent of that observed in post-traumatic trigeminal-autonomic cephalalgia. This framework opens new and promising perspectives on the understanding and medical management of ASI.

Keywords: tinnitus and hyperacousis, otalgia, pain, trigeminal nerve, acoustic shock, inflammation, referred pain

\section{INTRODUCTION}

Acoustic shocks are brief exposure to loud sounds that do not cause substantial hearing loss but can trigger a cluster of debilitating symptoms, i.e., otalgia, ear fullness, ear tension, tinnitus, sound intolerance, dizziness and head, face or neck aches $(1,2)$. In most cases, these symptoms are temporary and disappear within a few hours or days following the acoustic incident. However, in certain cases, they can become chronic and seriously affect quality of life (1). The pathophysiological mechanisms underlying these symptoms remain unknown, even though some authors have hypothesized a dysfunction in the tensor tympani muscle (TTM) $(1,2)$. The patient described here was able to precisely report his symptoms, their temporal evolution, and take pictures of his eardrums over time during symptom severity fluctuations. The psychoacoustic characteristics of his tinnitus and the functional integrity of the middle ears were also investigated. This invaluable dataset provides critical insights into the pathophysiology of the acoustic shock injury (ASI) and beyond, i.e., tinnitus, hyperacusis, and otalgia.

\section{METHODS}

The patient (NC) was a 27-year-old Caucasian male working as a general medical practitioner at the time of the acoustic shock. Written informed consent was obtained from the participant for the publication of this case report. On November 10th 2013, in a leisure shooting stand, without wearing any hearing protection, he was exposed to a unique and unexpected gunfire at an 
approximate $7 \mathrm{~m}$ distance from his right side. As bothersome symptoms progressively emerged after this acoustic incident, he attended several ENT clinics. Audiograms at week 3 and 7 after ASI (thresholds $<15 \mathrm{dBHL}$ at all tested frequencies from 0.125 to $8 \mathrm{kHz}$ ) and all other exams (tympanometry, blood check, and cerebral MRI) were normal. Later, he was asked to assess the severity of his symptoms, using a $0-10$ visual analog scale, for each ear several times a day (from May 1st to May 9th 2015). The symptoms were tingling, otalgia, ear tension, tinnitus loudness, neck pain/tension, temporal pain/tension, and pain/blocked nose. He also managed to photograph his eardrums using a video-otoscope (Firefly DE500 v1.1, $4 \mathrm{~mm}$ speculum). For analysis purpose, the images were transformed into numeric values estimating the "redness" of the eardrum. The number of red pixels was normalized according to the eardrum surface captured by the video-otoscope. In March 2017, NC visited our laboratory in Marseille to assess the psychoacoustic properties of his tinnitus and explored the middle ear function (Multifrequency Tympanometer Zodiac, Otometrics). Static admittance was obtained at four frequencies $(226,678,800$, and $1,000 \mathrm{~Hz}$ ). Admittance variation while the patient voluntarily (but not forcefully) eye blinked was investigated over time both at the pressure at which the admittance is maximal and at $\pm 50 \mathrm{daPa}$ from this value. Symptom severity from each ear was analyzed using principal component analysis (PCA). The PCA was performed on all symptoms, excluding neck and temporal pain as these symptoms did not affect the left side.

\section{RESULTS}

In regard to the clinical course, immediately after ASI, the patient felt a clicking in the right ear and thereafter reported a subjective perception of ear tension and fullness. At week 2 emerged a bilateral high-pitched fluctuating tinnitus. These auditory symptoms were associated with an erratic acute pain (sting or electrical shock) located deep in either ear. More than 3 weeks after the acoustic shock, the patient reported additional painful sensations starting around the concha and irradiating to the midface (constriction, blocked nose, and clear nasal discharge) and to the temporal region or the neck. Interestingly, the laterality and severity of the pain was correlated with the amount of tension perceived in the ipsilateral (right) ear. The psychoacoustic properties of the tinnitus, its amplitude modulation (tremolo), also varied according to the ear tension level. From low to mid tension, the tremolo varied from low frequency ("morse code") to high frequency ("cricket-like sound"). For high tension, tinnitus was described as a high-pitched whistling. The pitch of this tinnitus was measured at $12 \mathrm{kHz}$ and the tremolo, estimated from an amplitude modulated stimulus at the tinnitus frequency, was found at $32 \mathrm{~Hz}$ (the severity of ear tension and tinnitus loudness were 4 and 3, respectively). The loudness of this high-pitched tinnitus is reported in Figure 1. The patient also reported a low-pitched tinnitus associated with a sensation of fluttering in the ear. The low-pitched tinnitus was enhanced when something (earplug, stethoscope) was inserted in the ear canal. The high- and low-pitched tinnitus could be absent when the ear was completely relaxed. In contrast the tinnitus was not modulated by forceful head and neck contractions (3).

The temporal evolution of symptoms severity (Figure 1) was investigated using PCA. The first principal component (48\% of the total variance) represents the severity of the symptoms and does not discriminate between them. On the other hand, the second principal component (18\% of the total variance) separates two symptom clusters, namely "tension," "blocked nose," and "tympanum redness" for symptom cluster 1 and "tingling," "tinnitus," and "otalgia" for symptom cluster 2 (Figure 2, upper panels). The feeling of pain in the temporal region and the neck (plotted on the first two PCA axes) represent a third group of symptoms (symptom cluster 3), different from cluster 1 and 2. The symptom cluster 3 is reported in the right side only, is moderate in severity, and is present only occasionally compared to the other symptoms. The PCA indicates that the correlation between symptom cluster 3 and both symptom cluster 1 and 2 is low. Figure 2 (middle panels) shows the multi-dimensional data (symptom severity) plotted on the new coordinate system defined by the first two principal components at each time point. The symptoms are usually more severe in the right ear than in the left ear, and the maximum severity is also greater in the right ear. Interestingly, the two symptom clusters are reported in the right ear, while only symptom cluster 1 is reported in the left ear. The temporal dynamics of symptom severity (first principal component) in the left and right ear (Figure 2, bottom panel) is also clearly anti-correlated across the two ears.

The sensation of tension in the ear was associated with tympanum hyperemia (Figure 1, also see Video S1 in Supplementary Material). This hyperemia was also present during jaw muscle contraction (hypothetically involving the mylohyoid muscle) and was associated with an increase of the high-pitch tinnitus (see Video S2 in Supplementary Material). This phenomenon was reported to be only present when the sensation of tension in the ear was high and coincidentally, the tympanum was always showing dilated vessels.

The tympanometry measurements demonstrated different middle ear function for the two ears (Figure 3). Indeed, for the right ear, the static admittance was larger (Figure 3, upper panel) and the resonant frequency (estimated from the frequency at which the susceptance is null) was abnormally low $(<678 \mathrm{~Hz})$ (Figure 3, middle panel). Moreover, the admittance was modulated by eyelid closure, especially in the right ear (Figure 3, bottom panel). Finally, the stapedial reflexes were present and clinically normal in both ears at $500 \mathrm{~Hz}$ and $4 \mathrm{kHz}$ at 95-100 dB HL.

\section{DISCUSSION}

This report completes and adds to previous studies on acoustic shock (1). First, the symptoms reported after the acoustic shock, including their temporal dynamics and characteristics, have been described both qualitatively and quantitatively. This case reports all the symptoms that are commonly described after ASI, except vertigo and/or dizziness (1), and additional symptoms (nasal congestion, rhinorrhea, and tympanum hyperemia). Second, the functional integrity of the middle ears was also investigated 


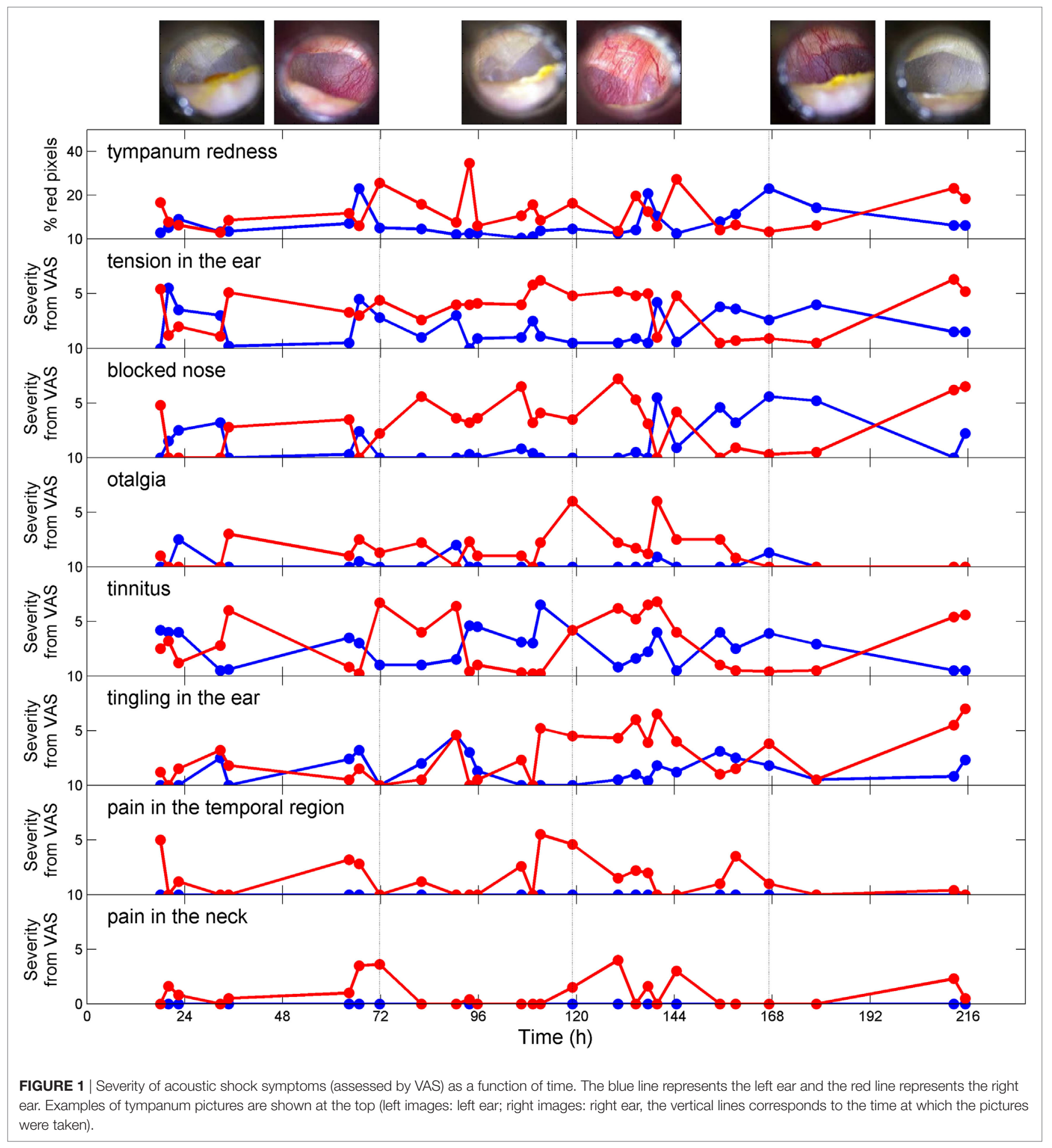

using multifrequency tympanometry and direct examination of the eardrums. Our findings provide further insights into the mechanisms involved in ASI, and in particular they question and extend the framework previously developed by others $(1,2)$.

This study is the first to provide experimental support suggesting that middle ear muscles (MEM) can behave abnormally after ASI. Indeed, middle ear admittance is changed by simple eyelid closure, eardrum movements in the right ear can be large enough to be visible by the naked eye and the fluttering sensation (likely accompanying MEM contraction) is enhanced by cutaneous stimulation of the ear canal (4-7). This abnormal behavior of MEM may result from a dysfunction and/or injury of MEM caused by an exaggerated contraction in response to the acoustic incident. While stapedius muscle contraction is activated by loud 

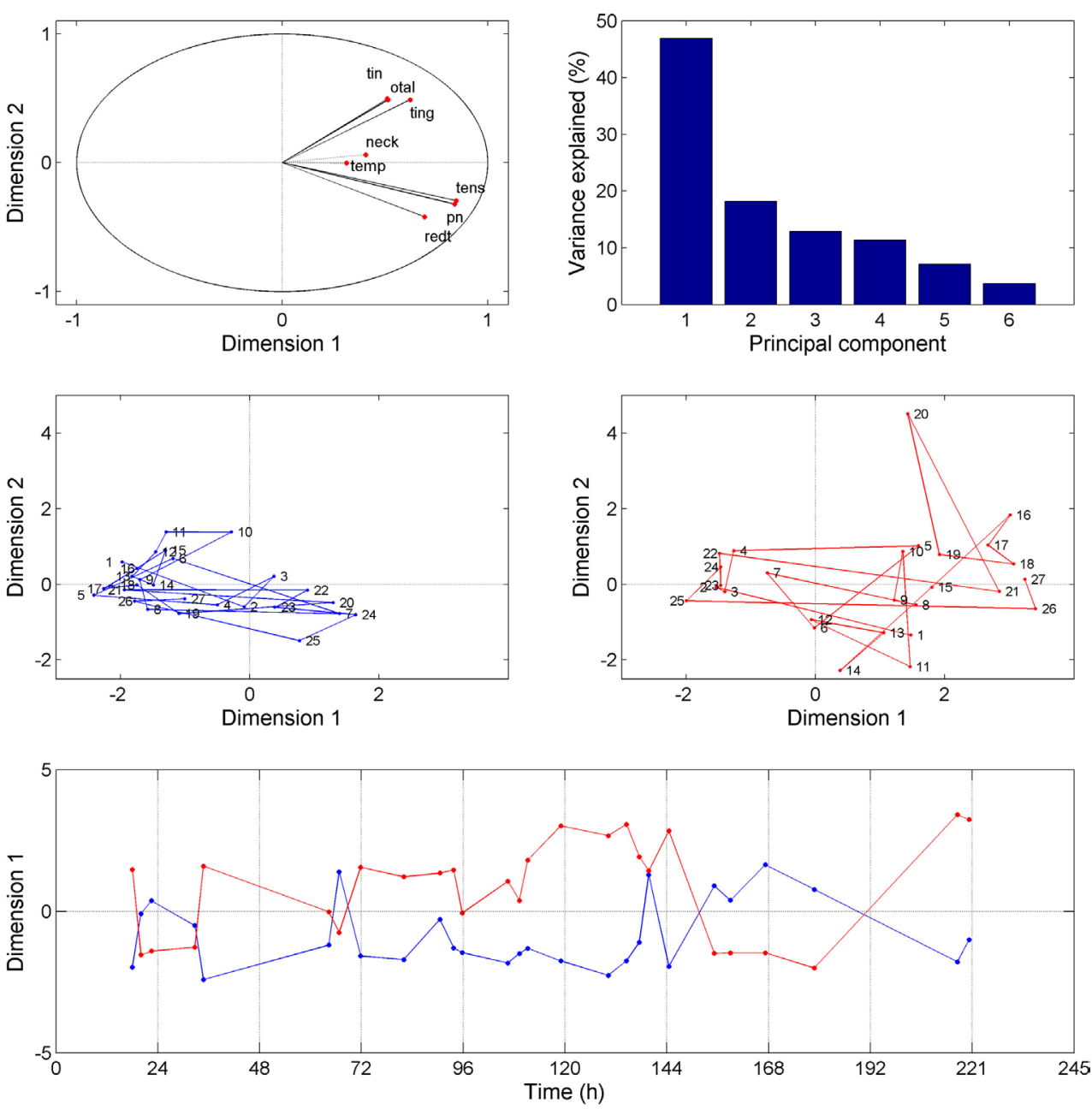

FIGURE 2 | Results obtained from the principal component analysis (PCA). The upper left panel represents each symptom as a function of the first two principal components derived from the PCA. The upper right panel shows the percentage of variance explained by the different principal components. The middle panels (left: left ear, right: right ear) show the symptoms plotted at each time point as a function the first two principal components. The bottom panel shows the first principal component for each ear (blue line: left ear, red line: right ear) as a function of time.

sounds, TTM contraction can be triggered by unexpected sounds through the general startle reflex (8). The low resonant frequency and large admittance in the most symptomatic (right) ear may reflect an abnormal ossicle arrangement, possibly caused by the exaggerated contraction of the MEM. The sensation of tension in the ear reported immediately after the acoustic shock may result from an abnormal contraction of the MEM signaled by the muscle spindles (9) and tympanum mechanoreceptors due to eardrum deformation (10). The traumatic acoustic episode may lead to anxiety and sensory hypersensitivity, which may in turn reduce the startle reflex threshold and/or potentiate the startle reflex (11), possibly through a descending serotoninergic innervation (12). This central feedback may facilitate tonic and phasic TTM contraction.

The putative exaggerated contraction of the MEM, eventually followed by tonic contraction, may be associated with MEM injury and inflammatory processes. In particular, the TTM is innervated by fibers containing substance $\mathrm{P}$ and calcitonin gene-related peptide, which may play a key role in the noiseinduced inflammation of the middle ear (13-15). Inflammation can then diffuse from the MEM to the tympanum and middle ear mucosa and can even become neurogenic as these tissues are rich in mastocytes (16-18). The diverse pain feelings in the ear (otalgia and tingling) likely result from middle ear inflammation and activation of the trigeminal nerve (TGN), which innervates the middle ear mucosa, the eardrum, and the TTM $(15,19)$. Intriguingly, tinnitus loudness is correlated to the severity of otalgia and tingling in the ear, suggesting that middle ear inflammation plays a role in tinnitus generation and/or severity. One can propose that antidromic activation of the TGN may change the vascular permeability of the blood vessels in the stria vascularis (20), which may eventually increase the endocochlear potential and produce tinnitus.

The MEM dysfunction/injury and the chronic inflammatory processes following ASI may be associated with neural hyperactivity in the trigeminal pathways. This neural hyperactivity may 

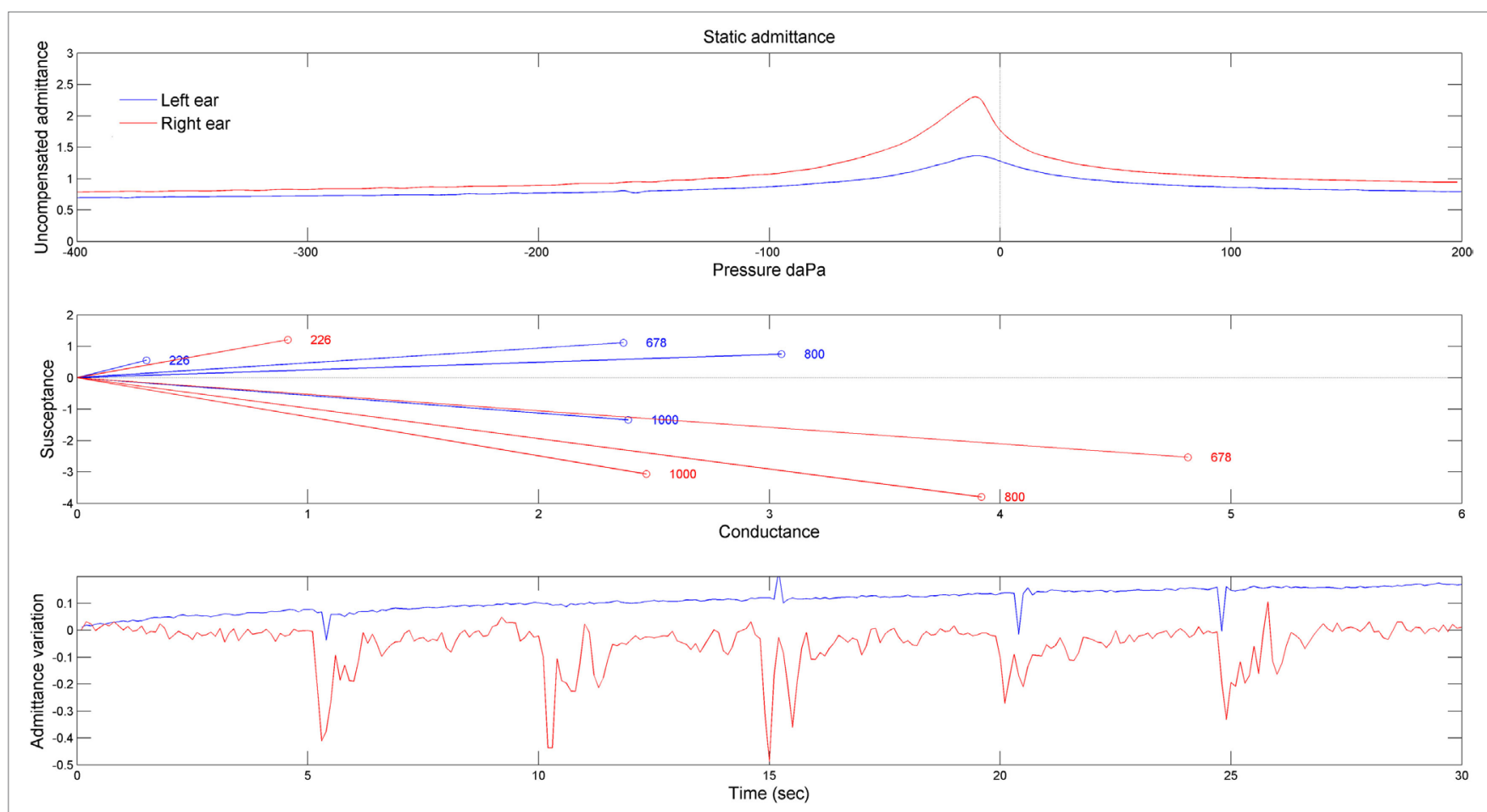

FIGURE 3 | Results obtained by tympanometry measurements. The upper panel shows the static admittance for the two ears obtained for a 226 Hz probe. The middle panel shows the susceptance as a function of the conductance at four frequencies of probe tone stimulation (226, 678,800 , and $1,000 \mathrm{~Hz}$ ). The bottom panel shows the admittance variation when the patient blinked voluntarily (but not forcefully) his eyes. The patient was asked to do so every $5 \mathrm{~s}$ (blue line: left ear, red line: right ear).

in turn lead to plastic changes (sensitization) at several levels of the trigeminal pathways (21). Pain in the neck and the temporal region may result from referred pain as a consequence of central sensitization, possibly at the trigemino-cervical complex (TCC) level where multimodal neurons are present (22). Sensitization may also contribute to shift the pathophysiology from acute to chronic (21).

Finally, the PCA suggests that the symptoms from cluster 1 originate from tightly coupled mechanisms. The trigeminal sensory inputs from regions of the head, including middle ear mucosa, eardrums, and the TTM $(14,15,19)$, are collected by the TCC that has a reflex connection with the superior salivatory nucleus (SSN). Interestingly, the SSN provides a parasympathetic innervation to the head region via the sphenopalatine ganglion. This reflex loop between the TGN and the parasympathetic pathway of the head has been suggested to account for the autonomic symptoms associated with trigeminal-autonomic cephalalgia (TAC) (23). The activation of the trigeminal-autonomic reflex in this case may account for the nasal congestion, rhinorrhea, and the tympanic hyperemia. The autonomic nervous system, which innervates the middle ear (18, 24-26), may also contribute to enhance the MEM tonus and the feeling of tension and aural fullness (27).

In summary, this case suggests that a complex pathophysiology can be involved in ASI. Initially, the acoustic shock may trigger an exaggerated MEM response causing muscle dysfunction/injury and inflammatory processes, which may later diffuse to the tympanum and middle ear mucosa. The activation of the trigeminal pathways due to inflammation may account for the diverse pain feelings in the ear and tinnitus (symptom cluster 2) and also for referred pain in the neck and the temporal region (symptom cluster 3 ) after induction of central sensitization. Finally, nasal congestion and tympanum hyperemia, which are tightly coupled to the feeling of ear tension (symptom cluster 1), may result from the activation of a trigeminal-autonomic reflex. Interestingly, the pathophysiology of ASI is strongly reminiscent of that of post-traumatic TAC (28). In this context, the case reported here may define a new clinical entity that we suggest calling post-traumatic trigeminal-autonomic otalgia. The clinical picture presented here, i.e., with numerous, diverse, and severe symptoms, could be an extreme form of this clinical entity and may be relatively rare. However, the prevalence of this clinical entity may be more prevalent than expected at first sight, as less symptomatic forms may also exist. Many patients with tinnitus and/or hyperacusis report one or more additional symptoms such as ear fullness, otalgia, tympanic flutter, and/or pain in the neck (2). This framework opens new and promising perspectives on the understanding and medical management of ASI and beyond, i.e., tinnitus and hyperacusis (23).

\section{ETHICS STATEMENT}

The case reported in the study gave written informed consent in accordance with the Declaration of Helsinki. 


\section{AUTHOR CONTRIBUTIONS}

AL, NC, DP, PF, LP, and AN collected data. LP and AN analyzed data and made figures. AN wrote a first version of the manuscript. All authors contributed to the final version of the manuscript.

\section{ACKNOWLEDGMENTS}

The authors would like to thank Dirk De Ridder, Anne Donnet, and Lénaic Monconduit for their useful comments on earlier versions of the manuscript. We also wish to thank Otometrics for their help and support. This work has been conducted with the

\section{REFERENCES}

1. Westcott M. Acoustic shock injury (ASI). Acta Otolaryngol Suppl (2006) 556:54-8. doi:10.1080/03655230600895531

2. Westcott M, Sanchez TG, Diges I, Saba C, Dineen R, McNeill C, et al. Tonic tensor tympani syndrome in tinnitus and hyperacusis patients: a multi-clinic prevalence study. Noise Health (2013) 15:117-28. doi:10.4103/1463-1741. 110295

3. Levine RA, Abel M, Cheng H. CNS somatosensory-auditory interactions elicit or modulate tinnitus. Exp Brain Res (2003) 153:643-8. doi:10.1007/ s00221-003-1747-3

4. Klockhoff IH, Anderson H. Recording of the stapedius reflex elicited by cutaneous stimulation; preliminary report. Acta Otolaryngol (1959) 50:451-4. doi:10.3109/00016485909129218

5. Ellenstein A, Yusuf N, Hallett M. Middle ear myoclonus: two informative cases and a systematic discussion of myogenic tinnitus. Tremor Other Hyperkinet Mov (N Y) (2013) 3. doi:10.7916/D8RX9BS1

6. Salomon G, Starr A. Electromyography of middle ear muscles in man during motor activities. Acta Neurol Scand (1963) 39:161-8. doi:10.111 1/j.1600-0404.1963.tb05317.x

7. Watanabe I, Kumagami H, Tsuda Y. Tinnitus due to abnormal contraction of stapedial muscle. An abnormal phenomenon in the course of facial nerve paralysis and its audiological significance. ORL J Otorhinolaryngol Relat Spec (1974) 36:217-26. doi:10.1159/000275177

8. Klockhoff I, Anderson H. Reflex activity in the tensor tympani muscle recorded in man; preliminary report. Acta Otolaryngol (1960) 51:184-8. doi:10.3109/00016486009124480

9. Kierner AC, Zelenka I, Lukas JR, Aigner M, Mayr R. Observations on the number, distribution and morphological peculiarities of muscle spindles in the tensor tympani and stapedius muscle of man. Hear Res (1999) 135:71-7. doi:10.1016/S0378-5955(99)00092-1

10. Nagai T, Tono T. Encapsulated nerve corpuscles in the human tympanic membrane. Arch Otorhinolaryngol (1989) 246:169-72. doi:10.1007/BF00456661

11. Grillon C. Models and mechanisms of anxiety: evidence from startle studies. Psychopharmacology (Berl) (2008) 199:421-37. doi:10.1007/ s00213-007-1019-1

12. Thompson AM, Thompson GC, Britton BH. Serotoninergic innervation of stapedial and tensor tympani motoneurons. Brain Res (1998) 787:175-8. doi:10.1016/S0006-8993(97)01020-2

13. Yamazaki M, Sato I. Distribution of substance P and the calcitonin gene-related peptide in the human tensor tympani muscle. Eur Arch Otorhinolaryngol (2014) 271:905-11. doi:10.1007/s00405-013-2469-1

14. Kierner AC, Mayer R, Adunka O. Is there a double innervation of the tensor tympani muscle in humans? Ann Otol Rhinol Laryngol (2003) 112:1056-8. doi: $10.1177 / 000348940311201211$

15. Uddman R, Grunditz T, Larsson A, Sundler F. Sensory innervation of the ear drum and middle-ear mucosa: retrograde tracing and immunocytochemistry. Cell Tissue Res (1988) 252:141-6. doi:10.1007/BF00213835

16. Ebmeyer J, Furukawa M, Pak K, Ebmeyer U, Sudhoff H, Broide D, et al. Role of mast cells in otitis media. J Allergy Clin Immunol (2005) 116:1129-35. doi:10.1016/j.jaci.2005.07.026 financial assistance of CNRS, Aix-Marseille Université, B2V, and the supplementary pension institution of Klesia.

\section{SUPPLEMENTARY MATERIAL}

The Supplementary Material for this article can be found online at http://journal.frontiersin.org/article/10.3389/fneur.2017.00420/ full\#supplementary-material.

VIDEO S1 | The video shows the right eardrum when the feeling of tension in the ear is high and when it is low.

VIDEO S2 | The video shows the right eardrum, when the tension in the ear is high, before, during, and after the mylohyoid muscle contraction.

17. Ylikoski J, Panula P. Neuropeptides in the middle ear mucosa. ORL J Otorhinolaryngol Relat Spec (1988) 50:176-82. doi:10.1159/ 000275987

18. Nagaraj BS, Linthicum FH. Autonomic innervation of the human middle ear: an immunohistochemical study. Am J Otolaryngol (1998) 19:75-82. doi:10.1016/S0196-0709(98)90099-0

19. Oyagi S, Ito J, Honjo I. The trigeminal sensory innervation to the middle ear, eustachian tube, and pharynx: a study by the horseradish peroxidase tracer method. Laryngoscope (1990) 100:873-7. doi:10.1288/00005537-19900800000014

20. Vass Z, Steyger PS, Hordichok AJ, Trune DR, Jancsó G, Nuttall AL. Capsaicin stimulation of the cochlea and electric stimulation of the trigeminal ganglion mediate vascular permeability in cochlear and vertebro-basilar arteries: a potential cause of inner ear dysfunction in headache. Neuroscience (2001) 103:189-201. doi:10.1016/S0306-4522(00)00521-2

21. Bernstein C, Burstein R. Sensitization of the trigeminovascular pathway: perspective and implications to migraine pathophysiology. J Clin Neurol (2012) 8:89-99. doi:10.3988/jcn.2012.8.2.89

22. Piovesan EJ, Kowacs PA, Oshinsky ML. Convergence of cervical and trigeminal sensory afferents. Curr Pain Headache Rep (2003) 7:377-83. doi:10.1007/ s11916-003-0037-x

23. Eller M, Goadsby PJ. Trigeminal autonomic cephalalgias. Oral Dis (2016) 22:1-8. doi:10.1111/odi.12263

24. Ogawa T, Rutka J. The presence of ganglion cells in the human middle ear: a histological survey. Acta Otolaryngol Suppl (1999) 540: 38-41.

25. Oyagi S, Ito J, Honjo I. The origin of autonomic nerves of the middle ear as studied by the horseradish peroxidase tracer method. Acta Otolaryngol (1987) 104:463-7. doi:10.3109/00016488709128275

26. Ito J, Oyagi S, Honjo I. Autonomic innervations in the middle ear and pharynx. Acta Otolaryngol Suppl (1993) 506:90-3. doi:10.3109/ 00016489309130249

27. Yuasa R, Kambayashi J, Saijo S, Hozawa K, Iino Y, Kaneko Y. Sensation of aural fullness and its treatment with an autonomic nerve blocking agent. Acta Otolaryngol Suppl (1987) 435:122-9. doi:10.3109/ 00016488709107361

28. Charney L, Rubino A, Cohen JM. A case of barotrauma-induced post-traumatic headache with a cluster headache phenotype. Headache (2016) 56:769-72. doi:10.1111/head.12727

Conflict of Interest Statement: The authors declare that the research was conducted in the absence of any commercial or financial relationships that could be construed as a potential conflict of interest.

Copyright (c) 2017 Londero, Charpentier, Ponsot, Fournier, Pezard and Noreña. This is an open-access article distributed under the terms of the Creative Commons Attribution License (CC BY). The use, distribution or reproduction in other forums is permitted, provided the original author(s) or licensor are credited and that the original publication in this journal is cited, in accordance with accepted academic practice. No use, distribution or reproduction is permitted which does not comply with these terms. 